\title{
Outerplanar Crossing Numbers, the Circular Arrangement Problem and Isoperimetric Functions
}

\author{
Éva Czabarka \\ Department of Mathematics \\ The College of William \& Mary \\ Williamsburg, VA 23187, USA \\ exczab@wm.edu \\ László A. Székely ${ }^{\dagger}$ \\ Department of Mathematics \\ University of South Carolina \\ Columbia, SC 29208, USA \\ szekely@math.sc.edu
}

\author{
Ondrej Sýkora* \\ Department of Computer Science \\ Loughborough University \\ Loughborough, Leicestershire LE11 3TU, UK \\ 0.Sykora@lboro.ac.uk \\ Imrich Vrto \\ Department of Informatics \\ Institute of Mathematics \\ Slovak Academy of Sciences \\ Dúbravská 9, 84104 \\ Bratislava, Slovak Republic \\ vrto@savba.sk
}

Submitted: Oct 31, 2003; Accepted: Nov 4, 2004; Published: Nov 12, 2004

Mathematics Subject Classifications: 05C62, 68R10

\begin{abstract}
We extend the lower bound in [15] for the outerplanar crossing number (in other terminologies also called convex, circular and one-page book crossing number) to a more general setting. In this setting we can show a better lower bound for the outerplanar crossing number of hypercubes than the best lower bound for the planar crossing number. We exhibit further sequences of graphs, whose outerplanar crossing number exceeds by a factor of $\log n$ the planar crossing number of the graph. We study the circular arrangement problem, as a lower bound for the linear arrangement problem, in a general fashion. We obtain new lower bounds for the circular arrangement problem. All the results depend on establishing good isoperimetric functions for certain classes of graphs. For several graph families new near-tight isoperimetric functions are established.
\end{abstract}

${ }^{*}$ This research was supported in part by the EPSRC grant GR/S76694/01.

${ }^{\dagger}$ This research was also supported in part by the NSF contracts Nr. 0072187 and 0302307.

†This research was supported in part by the VEGA grant No. 02/3164/23 


\section{Introduction}

This paper is a sequel to our paper with Shahrokhi [15]. We use similar notation as in that paper: $G=(V(G), E(G))$ denotes a graph and $d_{v}$ denotes the degree of $v \in V$. A drawing of $G$ is a placement of the vertices into distinct points of the plane and a representation of edges $u v$ by simple continuous curves connecting the corresponding points and not passing through any point corresponding to a vertex other than $u$ and $v$. A crossing is a common interior point of two edges of $G$. We also assume that any two curves representing the edges of $G$ have at most one interior point in common and that two curves incident to the same vertex do not cross. Let $\operatorname{cr}(G)$ denote the crossing number of $G$, i.e. the minimum number of crossings over all possible drawings of $G$ in the plane with the above properties (see [14] or [20]).

An important application area of crossing numbers is automated graph drawing. We know that the number of crossings influences the aesthetical properties and readability of graphs $[6,12]$.

An outerplanar (also called circular or convex) drawing of $G$ places the vertices on a circle and draws the edges as straight-line segments. The outerplanar crossing number of $G$ is the minimum number of pairs of crossing edges over all outerplanar drawings of $G$.

Let $\nu_{1}(G)$ denote the outerplanar [10] crossing number of $G$. There are other notations and terminologies used for this quantity. In [15] we used the term convex crossing number and notation $\operatorname{cr}^{*}(G)$. As the outerplanar drawing is topologically equivalent to the one-page drawing, we returned to the one-page book crossing number notation $\nu_{1}(G)$ in accordance with [14].

In our paper with Shahrokhi [15] we showed the following upper bound for the outerplanar crossing number through a divide-and-conquer algorithm:

Theorem 1. $\nu_{1}(G)=O\left(\left(\operatorname{cr}(G)+\sum_{v \in V} d_{v}^{2}\right) \log |V|\right)$.

We also showed on the example of the grid $P_{n} \times P_{n}$, where $P_{n}$ is the path on $n$ vertices, that Theorem 1 is the best possible, since $\nu_{1}\left(P_{n} \times P_{n}\right)=\Theta\left(n^{2} \log n\right)$. This example hinges on a general lower bound for $\nu_{1}(G)$ that we are going to present now.

We say that $f(x)$ is an isoperimetric function for $G$, if for any $k$-vertex subset $U$ of $V$ and $k \leq|V| / 2$, there are at least $f(k)$ edges between $U$ and $V \backslash U$. We require that $f(0)=0$. Define the difference function of $f$, denoted by $\Delta f$ as

$$
\Delta f(i)=f(i+1)-f(i)
$$

for any $i=0,1, \ldots,\left\lfloor\frac{|V|}{2}\right\rfloor-1$, and set

$$
\Delta^{2} f(i)=(\Delta(\Delta f))(i)=\Delta f(i+1)-\Delta f(i)
$$

for any $i=0,1, \ldots,\left\lfloor\frac{|V|}{2}\right\rfloor-2$. In [15] we have found the following lower bound for the outerplanar crossing number. 
Theorem 2. Assume that $f(x)$ is an isoperimetric function for $G=(V, E),|V|=n$ and $\Delta f$ is non-negative and decreasing till $\left\lfloor\frac{n}{2}\right\rfloor-1$. Then

$$
\nu_{1}(G) \geq-\frac{n}{8} \sum_{j=0}^{\left\lfloor\frac{n}{2}\right\rfloor-2} f(j) \Delta^{2} f(j)-\frac{1}{2} \sum_{v \in V} d_{v}^{2} .
$$

In this paper we show an extension of Theorem 2 (Theorem 3), which allows three different kind of improvement on Theorem 2. We will make use of all of them in Section 6. In particular, one extension relaxes the condition that $\Delta(f)$ is decreasing till $\left\lfloor\frac{|V|}{2}\right\rfloor-1$. This extension enables us to establish a near tight lower bound for the outerplanar crossing number of the hypercube. Note that Theorem 2 cannot be applied to the hypercube, since its isoperimetric function is not increasing till $\lfloor|V| / 2\rfloor-1$. We also show in Subsections 6.1 and 6.5 that even if the isoperimetric function is increasing till this point, our extension can improve the lower bound of Theorem 2 by a constant multiplicative factor.

In addition to the square lattice $P_{n} \times P_{n}$, [15] showed that "fat finite chunks" of the hexagonal lattice graph also have a logarithmic gap between $\operatorname{cr}(G)+\sum_{v} d_{v}^{2}$ and the outerplanar crossing number. The proof in [15] traced back this problem to that of the square lattice by ad hoc methods. Now we give a more direct proof using Theorem 2 , by establishing isoperimetric functions.

In [15] the logarithmic gap between $\operatorname{cr}(G)+\sum_{v} d_{v}^{2}$ and the outerplanar crossing number was shown only for sparse graphs. We provide here two families of dense graphs (Theorems 11, 12) with the same logarithmic gap. The basic tool is providing new isoperimetric functions for these graphs (Theorems 7,8 ).

We study the circular arrangement problem, which sets a lower bound for the usual linear arrangement problem. Recall that the linear arrangement problem requires the placement of the vertices of the graph into integer positions, and minimizes the sum of edge lengths over all placements. The circular arrangement problem requires the placement of the vertices of the graph into equidistant positions on a circle of perimeter $|V(G)|$, and minimizes the sum of lengths of paths on the circle, into which the edges of the graph $G$ are embedded on the circle, over all placements; and edges are embedded onto the shorter side of the circle. The circular arrangement problem has been introduced recently by Ching-Jung Guu [3], who solved in his thesis the circular arrangement problem for the hypercube, and Bezrukov and Schroeder [1], who showed that for trees the solutions for the linear arrangement problem and the circular arrangement problem are the same. The generalized F-linear arrangement and generalized F-circular arrangement problems assume a given function $F(x)$, and instead of summing up edge length, sum up $F(x)$ evaluated at the edge lengths. Probably the first occurence of the generalized $F$-linear arrangement problem was in the paper of Crimmins, Horwitz, and Palermo [5], who solved this problem in the case of $F(x)=x^{2}$ for the hypercube. Juvan and Mohar [9] studied the generalized $F$-linear arrangement problem for $F(x)=x^{p}$ for $p>0$, and in particular for $p=1,2$, and developed heuristics.

We show how to adapt our method of Theorems 2 and 3 to prove new lower bounds for the circular arrangement problem. These lower bounds are particularly good when 
$F(x)$ is near $x^{1 / 2}$.

\section{A Better Lower Bound}

Theorem 3. Assume that we have a family of graphs $G=G_{n}$ on $n$ vertices for infinitely many $n$ such that $f(x)=f_{n}(x)$ is an isoperimetric function for $G=(V, E), f(0)=0$ and $f(x)>0$ for $x \geq 1$. For the sequences $0 \leq s=s(n) \leq\left\lfloor\frac{n}{2}\right\rfloor-1$ and $0 \leq s_{0}=s_{0}(n) \leq s(n)$, assume that $\Delta f$ is non-negative and decreasing till $s$ and for each $s \leq x \leq\left\lfloor\frac{n}{2}\right\rfloor$ we have $f(x) \geq f\left(s_{0}\right)$. Define

$$
m_{f, s}(l)=\frac{f(l)}{f\left(\min \left\{\left\lfloor\frac{l}{2}\right\rfloor+1, s+1\right\}\right)} \quad \text { and } \quad \kappa\left(f, s_{0}, s\right)=\min _{s_{0} \leq l \leq\left\lfloor\frac{n}{2}\right\rfloor} m_{f, s}(l) .
$$

Assume that $\kappa=\kappa_{n}$ has a universal upper bound as $n \rightarrow \infty$, and in addition, as $n \rightarrow \infty$,

$$
n s_{0}=o(|E(G)|) \quad \text { or } \quad f\left(s_{0}\right)|E(G)|=o\left(\sum_{\substack{u v \in E: \\ l(u, v) \geq s_{0}}} f(l(u, v))\right) .
$$

Then we have

$$
\nu_{1}(G) \geq-\kappa\left(f, s_{0}, s\right)(1-o(1)) \cdot \frac{n}{8} \sum_{j=1}^{s-1} f(j) \Delta^{2} f(j)-\frac{1}{2} \sum_{v \in V} d_{v}^{2} .
$$

Proof. We follow the proof in [15] and modify it where it is necessary. Let $D$ be a outerplanar drawing of $G$. Without loss of generality we may assume that vertices in $D$ are placed on the perimeter of the unit circle in equidistant positions. Label the vertices by $0,1,2, \ldots, n-1$ according to their cyclic order. For simplicity, we will often identify a vertex with its corresponding integer. For $u, v \in V$ define the distance $l(u, v)$ between them by

$$
l(u, v)=\min \{|u-v|, n-|u-v|\} .
$$

Let us observe that (3) implies that

$$
\begin{aligned}
\sum_{\substack{u v \in E: \\
l(u, v)<s_{0}}} f(l(u, v)) \leq \sum_{\substack{u v \in E: \\
l(u, v)<s_{0}}} f\left(s_{0}\right) & \leq f\left(s_{0}\right) \min \left(n s_{0},|E(G)|\right) \\
& =o\left(\sum_{\substack{u v \in E \\
l(u, v) \geq s_{0}}} f(l(u, v))\right),
\end{aligned}
$$

where the last equality follows from the fact that for $x \geq s_{0}$ we have $f(x) \geq f\left(s_{0}\right)$.

For any $u v \in E$, let $c(u, v)$ denote the number of crossings of the edge $u v$ with other edges in $D$, and observe, as in [15], that $c(u, v) \geq f(l(u, v))-d_{u}-d_{v}$. Let $c(D)$ denote 
the number of crossings in the drawing $D$. We conclude that

$$
\begin{aligned}
c(D) & =\frac{1}{2} \sum_{u v \in E} c(u, v) \geq \frac{1}{2} \sum_{u v \in E}\left(f(l(u, v))-d_{u}-d_{v}\right) \\
& =\frac{1}{2} \sum_{u v \in E} f(l(u, v))-\frac{1}{2} \sum_{v \in V} d_{v}^{2} .
\end{aligned}
$$

We say that edge $u v \in E$ in the drawing $D$ covers a vertex $i$ if the unique shortest path between $u$ and $v$ (using only the edges on the boundary of the convex $n$-gon) contains $i$. If the shortest path is not unique (this happens if $n=2 l(u, v)$ ), then we pick arbitrarily one of the two shortest paths, and declare its vertices be covered by the $u v$ edge. (Note that an edge covers its endpoints.) For any edge $e=u v$ and any vertex $i$ define $l o a d_{u, v}(i)$, as

$$
\operatorname{load}_{u, v}(i)= \begin{cases}\Delta f(\min \{l(u, i), l(i, v)\}) & \text { if } e \text { covers } i \\ 0 & \text { otherwise. }\end{cases}
$$

Let $i \in V$. For $0 \leq t, E_{i, t}$ to be the set of all edges $u v \in E$ covering vertex $i$ in $D$ such that $\min \{l(i, u), l(i, v)\} \leq t$. Observe that $E_{i, j-1} \subseteq E_{i, j}$. Note that for any $i \in V$, and any $u v \in E_{i, j} \backslash E_{i, j-1}$, we have that $i$ is at distance $j$ from one of $u$ and $v$, and at distance at least $j$ from the other one. Therefore, for any $i \in V$, and any $u v \in E_{i, j} \backslash E_{i, j-1}$, we have $\operatorname{load}_{u, v}(i)=\Delta f(j)$, according to the definition of the load. Let $k_{t}$ denote $\sum_{i \in V}\left|E_{i, t}\right|$.

It is easy to see that for any $u v \in E$

$$
\sum_{i \in V: u v \in E_{i, s}} \operatorname{load}_{u, v}(i) \leq 2 \sum_{j=0}^{\min \left(\left\lfloor\frac{l(u, v)}{2}\right\rfloor, s\right)} \Delta f(j)=2 f\left(\min \left(\left\lfloor\frac{l(u, v)}{2}\right\rfloor, s\right)+1\right) .
$$

(The inequality uses the fact that $\Delta f \geq 0$ till $s$.)

We have

$$
\begin{aligned}
\sum_{u v \in E} \sum_{i:} \operatorname{load}_{u, v}(i) & =\sum_{i \in E_{i, s}} \sum_{j=0}^{s} \sum_{u v \in E_{i, j} \backslash E_{i, j-1}} \operatorname{load}_{u, v}(i) \\
& =\sum_{i \in V} \sum_{u v \in E_{i, 0}} \operatorname{load}_{u, v}(i)+\sum_{j=1}^{s} \sum_{i \in V} \sum_{u v \in E_{i, j} \backslash E_{i, j-1}} \operatorname{load}_{u, v}(i) \\
& =k_{0} \Delta f(0)+\sum_{j=1}^{s}\left(k_{j}-k_{j-1}\right) \Delta f(j),
\end{aligned}
$$

where the last equality is obtained by observing that the number of terms in the sum $\sum_{i \in V} \sum_{u v \in E_{i, j} \backslash E_{i, j-1}} \operatorname{load}_{u, v}(i)$, is $k_{j}-k_{j-1}$. It follows that

$$
\sum_{u v \in E} 2 f\left(\min \left(\left\lfloor\frac{l(u, v)}{2}\right\rfloor, s\right)+1\right) \geq \sum_{j=0}^{s-1} k_{j}(\Delta f(j)-\Delta f(j+1))+k_{s} \Delta f(s) .
$$


Note that up to (10) we did not use the assumption that $\Delta f$ is decreasing, we used only the fact that $\Delta f$ is non-negative till $s$. Since $k_{s} \Delta f(s) \geq 0$, we can drop the last term from the lower bound in (10). We also argue as in [15] - that for all $j \leq n / 2$,

$$
k_{j} \geq \frac{1}{2} n f(j) .
$$

To see this, consider any $j$ consecutive integers $i, i+1, \ldots, i+j-1$. Then at least $f(j)$ edges leave this $j$-set, and those edges must cover either $i$ or $i+j-1$. We may have counted some cases twice, since a vertex $i$ is an endpoint of two intervals of $j$, and if an edge goes from the first interval to the second, then this edge is counted twice covering $i$.

Definition (2) implies that for $l(u, v) \geq s_{0}$

$$
f\left(\min \left(\left\lfloor\frac{l(u, v)}{2}\right\rfloor, s\right)+1\right) \leq \frac{1}{\kappa\left(f, s_{0}, s\right)} f(l(u, v)) .
$$

Combining (6), (12), and the universal boundedness of $\kappa=\kappa_{n}$, we obtain

$$
\sum_{u v \in E} f\left(\min \left(\left\lfloor\frac{l(u, v)}{2}\right\rfloor, s\right)+1\right) \leq \frac{1+o(1)}{\kappa\left(f, s_{0}, s\right)} \sum_{u v \in E} f(l(u, v)) .
$$

We conclude using (10), (11), (13) and (7), that

$$
\begin{aligned}
-\frac{n}{2} \sum_{j=0}^{s-1} f(j) \Delta^{2} f(j) & \leq 2 \sum_{u v \in E} f\left(\min \left(\left\lfloor\frac{l(u, v)}{2}\right\rfloor, s\right)+1\right) \\
\leq \frac{2+o(1)}{\kappa\left(f, s_{0}, s\right)} \sum_{u v \in E} f(l(u, v)) & \leq \frac{4+o(1)}{\kappa\left(f, s_{0}, s\right)}\left(c(D)+\frac{1}{2} \sum_{v} d_{v}^{2}\right) .
\end{aligned}
$$

Note that in the first inequality we used (11) and the condition that $\Delta f(j)-\Delta f(j+1) \geq 0$, i.e. that $\Delta f$ is decreasing till $s$. This, together with the fact that $f(0)=0$, finishes the proof.

Of course, the choice $s_{0}=0$ is always possible, but then $l=1$ in (2) does not allow to have $\kappa>1$, which is our goal to obtain improvement over (1) by (4). Alternatively, if $f(x)$ is a smooth function, one can get a more convenient estimation than in Theorem 3 .

Theorem 4. Under the assumptions of Theorem 3, with additional assumptions that for an $s \leq\left\lfloor\frac{n}{2}\right\rfloor-2$ on the interval $(0, s+1) f^{\prime}$ and $f^{\prime \prime}$ exist, $f^{\prime} \geq 0, f^{\prime \prime} \leq 0$ and increasing, one can change the right hand side of (4) to

$$
\nu_{1}(G) \geq-\frac{n}{8} \kappa\left(f, s_{0}, s\right)(1-o(1)) \int_{0}^{s-1} f(x) f^{\prime \prime}(x+3) d x-\frac{1}{2} \sum_{v \in V} d_{v}^{2} .
$$


Proof. Recall Taylor's formula with remainder:

$$
\begin{aligned}
f(j+2)= & f(j+1)+f^{\prime}(j+1)+\frac{1}{2} f^{\prime \prime}(\xi) \quad(j+1<\xi<j+2), \\
f(j)= & f(j+1)-f^{\prime}(j+1)+\frac{1}{2} f^{\prime \prime}(\eta) \quad(j<\eta<j+1), \\
& \Delta f(j)-\Delta f(j+1)=-\frac{1}{2}\left(f^{\prime \prime}(\xi)+f^{\prime \prime}(\eta)\right)>-f^{\prime \prime}(j+2) .
\end{aligned}
$$

Now

$$
-\sum_{j=1}^{s-1} f(j) \Delta^{2} f(j) \geq-\sum_{j=1}^{s-1} f(j) f^{\prime \prime}(j+2),
$$

and for any $1 \leq j \leq s-1$, one has

$$
-f(j) f^{\prime \prime}(j+2) \geq-\int_{j-1}^{j} f(x) f^{\prime \prime}(x+3) d x .
$$

(14) is easily obtained from (4) using (15), and (16).

Even if $f(x)$ is not as smooth as required above, Theorem 4 still applies with slight modification, if we allow appropriate error terms arising at the "bad" points of $f(x)$. There are many ways to handle this problem. We may relax the additional assumptions of Theorem 4 as follows. $f^{\prime}$ and $f^{\prime \prime}$ may be undefined in a bounded number of points in $(0, s+1)$, but $f^{\prime} \geq 0, f^{\prime \prime} \leq 0$ and $f^{\prime \prime}$ must be increasing in each of the subintervals between the special points. Assume that $\max _{[1, s+1]}\left|f_{n}(x) \Delta^{2} f_{n}(x)\right|$ and $\max _{[1, s+1]}\left|f_{n}(x) f_{n}^{\prime \prime}(x+3)\right|$ are little-oh the right-hand side of (14) as $n \rightarrow \infty$. Then Theorem 4 still holds. This relaxation of Theorem 4 allows to prove Theorems 11 and 12 by integrating the piecewise smooth isoperimetric functions from Theorems 7 and 8 . Similar relaxation can be given for the conditions of Theorem 5 as well.

\section{Circular Arrangement Problem}

We define the generalized $F$-linear arrangement problem as follows. Let us be given a non-negative and increasing real function $F(x)$. Let $h$ be a bijection between $V(G)$ and the set of integers $\{1,2, \ldots,|V|\}$. Define the generalized $F$-linear arrangement value as

$$
L_{F}(h, G)=\sum_{u v \in E(G)} F(|h(u)-h(v)|) .
$$

The generalized F-linear arrangement problem asks for

$$
L_{F}(G)=\min _{h} L_{F}(h, G)=\min _{h} \sum_{u v \in E(G)} F(|h(u)-h(v)|) .
$$

We define similarly the generalized $F$-circular arrangement problem as follows. Let us be given a non-negative and increasing real function $F(x)$. Let $h$ be a bijection between 
$V(G)$ and points $\{1,2, \ldots,|V|\}$ placed equidistantly on a circle in this order. Define the generalized $F$-circular arrangement value as

$$
L_{F}^{o}(h, G)=\sum_{u v \in E(G)} F(l(h(u), h(v))),
$$

where $l$ is the distance function defined in (5). The generalized F-linear arrangement problem asks for

$$
L_{F}^{o}(G)=\min _{h} L_{F}^{o}(h, G)=\min _{h} \sum_{u v \in E(G)} F(l(h(u), h(v))) .
$$

It is clear that $L_{F}^{o}(h, G) \leq L_{F}(h, G)$, and consequently $L_{F}^{o}(G) \leq L_{F}(G)$. Therefore, it is of interest to set lower bounds on the generalized $F$-circular arrangement problem.

Theorem 5. Assume that we have a family of graphs $G=G_{n}$ on $n$ vertices for infinitely many $n, f(x)=f_{n}(x) \geq 0$ is an isoperimetric function for $G=(V, E)$. Assume that $F(x)>0$ for $x \geq 1$ and $F(0)=0$, a function not dependent on $n$. For the sequences $0 \leq s=s(n) \leq\left\lfloor\frac{n}{2}\right\rfloor-1$ and $0 \leq s_{0}=s_{0}(n) \leq s(n)$, assume that $\Delta F$ is non-negative and decreasing till $\infty$. Define

$$
m_{F, s}(l)=\frac{F(l)}{F\left(\min \left\{\left\lfloor\frac{l}{2}\right\rfloor+1, s+1\right\}\right)} \quad \text { and } \quad \kappa\left(F, s_{0}, s\right)=\min _{s_{0} \leq l \leq\left\lfloor\frac{n}{2}\right\rfloor} m_{F, s}(l) .
$$

Assume that $\kappa=\kappa_{n}$ has a universal upper bound as $n \rightarrow \infty$, and in addition, as $n \rightarrow \infty$, $n s_{0}=o(|E(G)|)$ or $F\left(s_{0}\right)|E(G)|=o\left(\sum_{\substack{u v \in E: \\ l(u, v) \geq s_{0}}} F(l(u, v))\right)$. Then we have

$$
L_{F}^{o}(G) \geq-\kappa\left(F, s_{0}, s\right)(1-o(1)) \cdot \frac{n}{4} \sum_{j=0}^{s-1} f(j) \Delta^{2} F(j) .
$$

Furthermore, with additional assumptions that for an $s(n) \leq\left\lfloor\frac{n}{2}\right\rfloor-2$, on the interval $(0, s+1) F^{\prime}$ and $F^{\prime \prime}$ exist, $F^{\prime} \geq 0, F^{\prime \prime} \leq 0$ and increasing, one can change the right hand side of (21) to

$$
-\kappa\left(F, s_{0}, s\right)(1-o(1)) \cdot \frac{n}{4} \int_{1}^{s-1} f(x) F^{\prime \prime}(x+3) d x .
$$

Proof. Mutatis mutandis, we follow the proof of Theorem 3. We define the load as in (8), but with $\Delta_{F}$ instead of $\Delta_{f}$. Formula (9) is substituted by

$$
\sum_{i \in V: u v \in E_{i, s}} \operatorname{load}_{u, v}(i) \leq 2 \sum_{j=0}^{\min \left\{\left\lfloor\frac{l(u, v)}{2}\right\rfloor, s\right\}} \Delta_{F}(j)=2 F\left(\min \left(\left\lfloor\frac{l(u, v)}{2}\right\rfloor, s\right)+1\right) .
$$

We obtain

$$
\sum_{u v \in E} F(l(u, v)) \geq \kappa\left(F, s_{0}, s\right)(1-o(1)) \sum_{u v \in E} F\left(\min \left(\left\lfloor\frac{l(u, v)}{2}\right\rfloor, s\right)+1\right)
$$


as we obtained (13). Using (23), the partial summation leading to (10), and formula (11) as it is, we obtain:

$$
\frac{1}{2} \sum_{u v \in E} \sum_{i \in V: u v \in E_{i, s}} \operatorname{load}_{u, v}(i) \geq-\frac{n}{4} \sum_{j=0}^{s-1} f(j) \Delta^{2} F(j) .
$$

Finally, (22) is easily obtained from (21) using Taylor's formula with remainder, like in the argument in the previous section.

\section{Citing Isoperimetric Inequalities}

It is clear that the complete graph $K_{n}$ has isoperimetric function

$$
f(x)=x(n-x) .
$$

For the hypercube $Q_{n}$ on $2^{n}$ vertices, Chung et al. [4] established the isoperimetric function

$$
f(x)=x\left(n-\log _{2} x\right) .
$$

Bollobás and Leader [2] established the isoperimetric function

$$
f(x)=\sqrt{2 x}
$$

for the $n \times n$ grid $P_{n} \times P_{n}$, i.e. the Cartesian product of two $n$-vertex paths $P_{n}$. More generally, they established the isoperimetric function $f(x)$

$$
f(x)= \begin{cases}\sqrt{2 x} & \text { if } x \leq \frac{1}{2} n^{2} \\ n & \text { if } \frac{1}{2} n^{2} \leq x \leq \frac{k n}{2}\end{cases}
$$

for the $n \times k$ grid $P_{n} \times P_{k}$, i.e. the Cartesian product of an $n$-vertex path with a $k$-vertex path, for $n \leq k$. Tillich [21] has made a substantial study of isoperimetric inequalities in Cartesian product graphs. For $K_{p}^{n}$, the $n$-th Cartesian power of the complete graph $K_{n}$, he proved

$$
f(x)=(p-1) x\left(n-\log _{p} x\right),
$$

which gives back the isoperimetric inequality (25) for the hypercube for $p=2$. For the Cartesian power $P^{n}$ of the Petersen graph $P$, Tillich [21] provided two isoperimetric functions that are incomparable:

$$
\begin{aligned}
& f_{1}(x)=2 x\left(5^{n}-x\right) / 5^{n} \\
& f_{2}(x)=2 x\left(n-\log _{5} x\right) .
\end{aligned}
$$

Recall the definition of the edge-forwarding index $\pi(G)$ of a graph $G$. For every ordered pair of vertices $(a, b)$, where $a \neq b \in V(G)$, assign a path of $G$ connecting $a$ to $b$. The congestion of an edge is the number of paths using this edge, and the congestion of the 
path system is the maximum congestion of edges. The edge-forwarding index $\pi(G)$ of the graph $G$ is the minimum congestion of any such path system. Now, we have immediately from the definition the following isoperimetric function:

$$
f(x)=\frac{2 x(n-x)}{\pi(G)},
$$

where $n$ is the number of vertices. Note that the edge-forwarding index is also studied under the name optimal integral concurrent multicommodity flow.

\section{$5 \quad$ Proving Relevant Isoperimetric Inequalities}

In this section we prove isoperimetric inequalities for one sparse and two dense graphs closely related to the grid. By applying Theorem 2 we get tight lower bounds in section 6 .

First, we are going to study a relative of the grid, which we call a rhombus of hexagons, see Fig. 1.

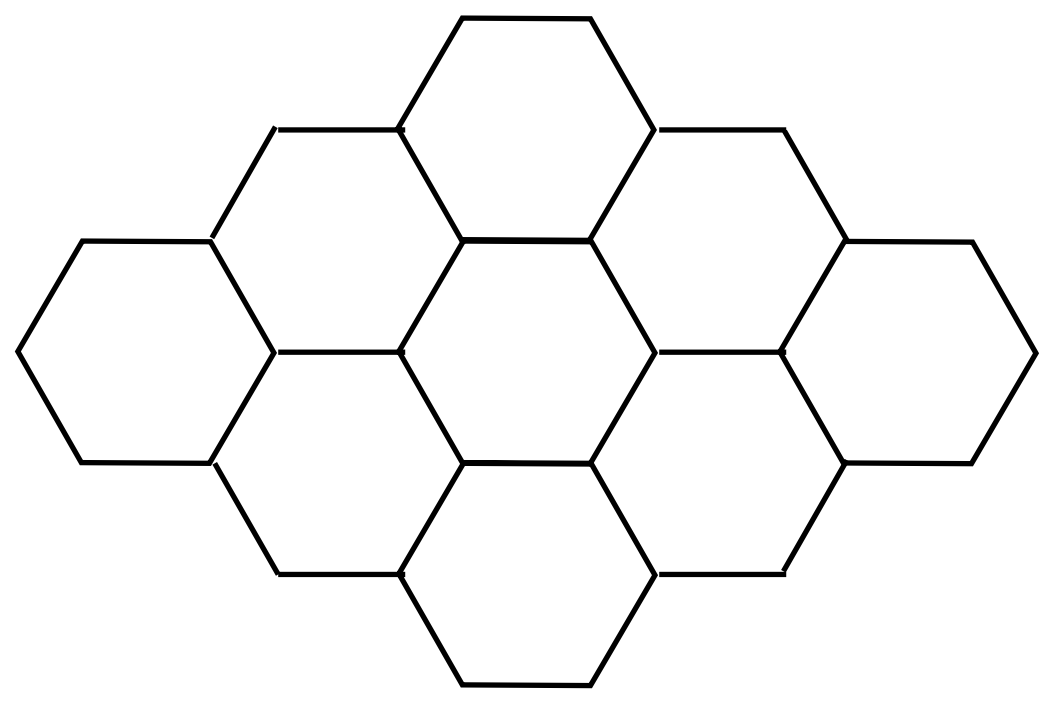

Figure 1: The $3 \times 3$ rhombus of hexagons.

Theorem 6. The following is an isoperimetric function for a rhombus of hexagons with $n>n_{0}$ vertices:

$$
f(x)=\frac{1}{3} \sqrt{x}
$$


Proof. Observe that by contracting all horizontal edges in the rhombus of hexagons, we obtain an ordinary square grid. With two exceptions at the corners of the grid, vertices of the square grid have two pre-images, and the exceptional vertices have just one.

Let us be given a subset $X$ of vertices of the rhombus of hexagons, with $x=|X|$, $x \leq n / 2$. Assume that $x=a+2 b+c$, where $a$ denotes the number of vertices from $X$ which occur alone on a horizontal edge, $b$ denotes the number of pairs of vertices from $X$ which occur on the same horizontal edge, and $c$ denotes the number of exceptional vertices from $X$.

If $a \geq \frac{1}{3} \sqrt{x}$, then we have $a$ horizontal edges between $X$ and $\bar{X}$ in the rhombus of hexagons, and we are at home. Therefore we may assume $a \leq \frac{1}{3} \sqrt{x} \leq \frac{1}{3} \sqrt{n / 2}$; and as we noted above, $c \leq 2$.

Let $X^{\prime}$ denote the set of vertices in the square grid, which have at least one pre-image in $X$. Clearly $\left|X^{\prime}\right|=a+b+c$. It is easy to check that edges between $X^{\prime}$ and $\overline{\left(X^{\prime}\right)}$ correspond to distinct edges between $X$ and $\bar{X}$, except in the case shown in Fig. 2, where 2 edges in the square grid correspond to a single edge in the rhombus of hexagons.
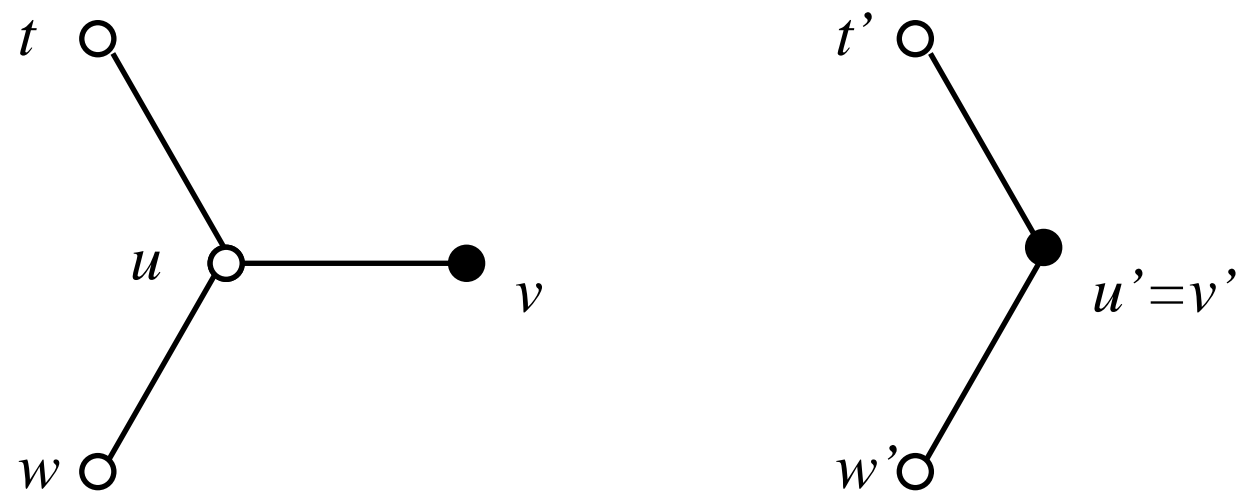

Figure 2: $t, u, w \in \bar{X}, v \in X ; t^{\prime}, w^{\prime} \in \overline{\left(X^{\prime}\right)}, u^{\prime}=v^{\prime} \in X^{\prime}$. A single edge in $E(X, \bar{X})$ may correspond to two edges in $E\left(X^{\prime}, \overline{\left(X^{\prime}\right)}\right)$.

Therefore, $|E(X, \bar{X})| \geq \frac{1}{2}\left|E\left(X^{\prime}, \overline{\left(X^{\prime}\right)}\right)\right|$. The square grid has $\frac{n}{2}+1$ vertices, and $\left|X^{\prime}\right| \leq \frac{n}{4}+\frac{1}{3} \sqrt{n / 2}+2$. Formula $(26)$ applies to $\left|E\left(X^{\prime}, \overline{\left(X^{\prime}\right)}\right)\right|$, if $\left|X^{\prime}\right| \leq \frac{n}{4}$; and since $\left|X^{\prime}\right|$ can only slightly exceed $\frac{n}{4}$, for sufficiently large $n$, a slightly weaker formula holds, reducing 2 to $9 / 5$ : $\left|E\left(X^{\prime}, \overline{\left(X^{\prime}\right)}\right)\right| \geq \sqrt{\frac{9}{5}(a+b+c)}$. Combining the observations yields $|E(X, \bar{X})| \geq \frac{1}{2} \sqrt{\frac{9}{5}(a+b+c)} \geq \frac{1}{2} \sqrt{\frac{9}{10} x} \geq \frac{1}{3} \sqrt{x}$.

Next we study the isoperimetric inequality of two graphs closely related to the grid. Let $P(n, k)$ denote the graph that we obtain from an $n$-vertex path by joining vertices whose distance in the path is at most $k$. Let $G(n, k)$ denote the Cartesian product $P(n, k) \times P(n, k)$. We will find an isoperimetric function for $G(n, k)$ in the case when $k$ is an even divisor of $2 n$. To set lower bounds, we first study the isoperimetric problem in $G\left(\frac{k}{2}, \frac{k}{2}\right)=K_{\frac{k}{2}} \times K_{\frac{k}{2}}$. 
Lemma 1. Let $0<c<1 / 2$ and $2 / 3 \leq d \leq 1$. We put the vertices of $K_{\frac{k}{2}} \times K_{\frac{k}{2}}$ into $\frac{k}{2}$ rows and $\frac{k}{2}$ columns in the natural way. For any $X \subset V\left(K_{\frac{k}{2}} \times K_{\frac{k}{2}}\right)$,

(i) If a row $S$ has the property, that $|X \cap S| \leq(1-c) \frac{k}{2}$, then $|E(X \cap S, \bar{X} \cap S)| \geq \frac{c k}{2}|X \cap S|$.

(ii) If at least $c|X|$ elements of $X$ fall into rows $S$ such that $|X \cap S| \leq(1-c) \frac{k}{2}$, then $|E(X, \bar{X})| \geq \frac{c^{2} k}{2}|X|$.

(iii) If $|X| \leq(1-2 c)^{3}\left(\frac{k}{2}\right)^{2}$, then $|E(X, \bar{X})| \geq \frac{c^{2} k}{2}|X|$.

(iv) If $|X| \leq \frac{1}{3}\left(\frac{k}{2}\right)^{2}$, then $|E(X, \bar{X})| \geq \frac{k}{86}|X|$.

(v) If $|X| \leq \frac{2}{3}\left(\frac{k}{2}\right)^{2}$, then $|E(X, \bar{X})| \geq \frac{k}{501}|X|$.

(vi) If $|X|=d\left(\frac{k}{2}\right)^{2}$, then $|E(X, \bar{X})| \geq \frac{k(1-d)}{86 d}|X|$.

Proof. (i) is trivial, (ii) is immediate from (i). Parts (iv) and (v) easily follow from (iii) by setting $c=\frac{1}{2}\left[1-(1 / 3)^{1 / 3}\right]$ and $c=\frac{1}{2}\left[1-(2 / 3)^{1 / 3}\right]$, respectively. To show (iii), assume that the conditions of (ii) fail for the rows as well as for the colums (otherwise $\left.|E(X, \bar{X})| \geq \frac{c^{2} k}{2}|X|\right)$. Then at least $(1-c)|X|$ vertices of $X$ fall into rows $S$ such that $|X \cap S| \geq(1-c) \frac{k}{2}$. Let $a$ denote the number of rows $S$ such that $|X \cap S| \geq(1-c) \cdot \frac{k}{2}$, and let $A$ denote the subset of elements of $X$ which lie on such rows. Similarly, at least $(1-c) \cdot|X|$ vertices of $X$ fall into columns $T$ such that $|X \cap T| \geq(1-c) \frac{k}{2}$. Let $b$ denote the number of columns $T$ with $|X \cap T| \geq(1-c) \frac{k}{2}$, and let $B$ denote the subset of elements of $X$ which lie on such columns. Clearly $|X| \geq(1-c) a \frac{k}{2}$ and $|X| \geq(1-c) b \frac{k}{2}$. Since the intersection of $a$ rows and $b$ columns contains $a b$ vertices, by inclusion-exclusion we have:

$$
|X| \geq|A|+|B|-a b \geq 2(1-c)|X|-\left(\frac{|X|}{(1-c) \frac{k}{2}}\right)^{2}
$$

which implies $|X| \geq(1-2 c)(1-c)^{2} \frac{k^{2}}{4}>(1-2 c)^{3} \frac{k^{2}}{4}$, a contradiction.

To show (vi), note that $\frac{1-d}{d}|X|=|X| \leq \frac{1}{3}\left(\frac{k}{2}\right)^{2}$, so from (iv) we obtain that $|E(X, \bar{X})| \geq$ $\frac{k(1-d)}{d 86}|X|$.

We also make use of the following rather technical result:

Lemma 2. Put the vertices of $K_{\frac{k}{2}} \times K_{k}$ into $\frac{k}{2}$ rows and $k$ columns in the natural way, and divide this graph into two disjoint $\frac{k}{2} \times \frac{k}{2}$ squares, $A$ and $B$. Let $X$ be a subset of the vertices such that $|X \cap A| \leq \frac{k^{2}}{6}$ and $|X \cap B|>\frac{k^{2}}{6}$. Then the number of $(X, \bar{X})$-type edges not entirely in $A$ is at least $\frac{k^{3}}{1032}$.

Proof. Let $d=|X \cap B| \cdot \frac{4}{k^{2}}$, then by our assumptions $\frac{2}{3}<d \leq 1$. By Lemma 1 (vi), $|E(X \cap B, \bar{X} \cap B)| \geq \frac{k^{3}(1-d)}{344}$. By construction, $A$ and $B$ share their rows. Let $x_{i}\left(y_{i}\right)$ denote the proportion of elements from $X$ in the $i^{\text {th }}$ row of $B(A)$. (With other words, the number of such elements is $\frac{k x_{i}}{2}$ and $\frac{k y_{i}}{2}$, respectively.) Then $\sum_{i=1}^{k / 2} x_{i}=d k / 2$, and $\sum_{i=1}^{k / 2} y_{i} \leq k / 3$. We use the estimate

$$
\sum_{i=1}^{k / 2} x_{i}\left(1-y_{i}\right) \geq \sum_{i=1}^{k / 2}\left(x_{i}-y_{i}\right) \geq(3 d-2) \cdot \frac{k}{6}
$$


and obtain that the number of $(X \cap B, \bar{X} \cap A)$ type edges is at least $\frac{k^{2}}{4} \sum_{i=1}^{k / 2} x_{i}\left(1-y_{i}\right) \geq$ $(3 d-2) \frac{k^{3}}{24}$. Therefore the number of $(X, \bar{X})$ type edges not entirely in $A$ is at least $\frac{k^{3}(1-d)}{344}+\frac{k^{3}(3 d-2)}{24}=(126 d-83) \frac{k^{3}}{1032} \geq \frac{k^{3}}{1032}$.

Theorem 7. Let $k$ be an even divisor of $2 n$. The following is an isoperimetric function for $G(n, k)$ :

$$
f(x)= \begin{cases}\frac{k}{501} \cdot x & \text { if } 0 \leq x \leq\left(\frac{501 k}{2064}\right)^{2}, \\ \frac{k^{2}}{2064} \cdot \sqrt{x} & \text { if }\left(\frac{501 k}{2064}\right)^{2} \leq x \leq n^{2} / 2 .\end{cases}
$$

Proof. We make a $(2 n / k) \times(2 n / k)$ grid $G^{\prime}$ by cutting the original grid $G$ into $(k / 2) \times(k / 2)$ squares. Let us be given a set $X$ of vertices of $G,|X|=x\left(0 \leq x \leq n^{2} / 2\right)$. We have to find $f(x)$ edges in $G(n, k)$ leaving the set $X$. Every $v \in X$ falls into some $(2 n / k) \times(2 n / k)$ square in $G^{\prime}$. Let us denote this square by $S_{v}$. Set the density of the square $S_{v}$ as $d\left(S_{v}\right)=\left|X \cap S_{v}\right| /\left|S_{v}\right|=4\left|X \cap S_{v}\right| / k^{2}$. We distinguish cases:

(i) $v$ is a small vertex, $\left(S_{v}\right.$ is a small square) if $d\left(S_{v}\right) \leq \frac{2}{3}$

(ii) $v$ is large vertex, $\left(S_{v}\right.$ is a large square) if $\frac{2}{3}<d\left(S_{v}\right)$.

Let $x_{S}\left(x_{L}\right)$ denote the number of small (large) vertices of $X$. We have $x=x_{S}+x_{L}$. It follows from Lemma $1(\mathrm{v})$ that the number of $(X, \bar{X})$ edges inside the small squares is at least $k x_{s} / 501=k\left(x-x_{L}\right) / 501$.

Let us estimate the number of large squares of $G^{\prime}$. Clearly their number $n^{\prime}$ falls between $x_{L} /(k / 2)^{2}$ and $3 x_{L} /\left[2(k / 2)^{2}\right]$. According to the isoperimetric inequality for grids (26), the number of (large square, small square) pairs that share a common side is at least $\sqrt{2 \min \left\{n^{\prime},(2 n / k)^{2}-n^{\prime}\right\}} \geq(2 / k) \cdot \sqrt{2 \min \left\{x_{L}, n^{2}-3 x_{L} / 2\right\}} \geq 2 \sqrt{x_{L}} / k$. Lemma 2 tells us that at each such pair of squares there is at least $\frac{k^{3}}{1032}$ edges of type $(X, \bar{X})$ that are not inside the small square. Observe that every large square shares side edges with at most 4 small squares. Therefore, the number $(X, \bar{X})$ type edges not in a small square is at least $\frac{1}{4} \cdot \frac{k^{2}}{516} \cdot \sqrt{x_{L}}$. Consequently, the number of $(X, \bar{X})$ type edges is at least

$$
T\left(x_{L}\right)=k \frac{x-x_{L}}{501}+\frac{k^{2} \sqrt{x_{L}}}{2064} .
$$

As $T\left(x_{L}\right)$ is quadratic in $\sqrt{x_{L}}$, it is easy to see that $T\left(x_{L}\right) \geq \min \{T(0), T(x)\} . T(0)=$ $k x / 501$ and $T(x)=k^{2} \sqrt{x} / 2064$, so $T(0) \leq T(x)$ when $x \leq \frac{501^{2} k^{2}}{2064^{2}}$, otherwise $T(0)>T(x)$, and so we arrived at (33).

Let $H(n, k)$ denote the graph that we obtain by taking the $n \times n$ grid $G$ in the plane, joining vertices whose distance is at most $k$

We establish an isoperimetric inequality for $H(n, k)$ when 3 divides $k$ and $k$ divides $3 n$. As before, we start with 2 lemmas.

Lemma 3. Let $0 \leq d \leq 1$. For any $X \subset V\left(K_{\frac{k}{3} \times \frac{k}{3}}\right)$,

(i) If $|X| \leq d\left(\frac{k}{3}\right)^{2}$, then $E(X, \bar{X})\left|\geq \frac{(1-d) k^{2}}{9}\right| X \mid$.

(ii) If $|X| \leq \frac{2}{3}\left(\frac{k}{3}\right)^{2}$, then $|E(X, \bar{X})| \geq \frac{k^{2}}{27}|X|$. 
Lemma 4. Arrange the vertices of $K_{\frac{k}{3} \times \frac{2 k}{3}}$ into $k / 3$ rows and $2 k / 3$ columns and divide them into two squares, $A$ and $B$. Let $X$ be a subset of the vertices such that $|X \cap A| \leq \frac{2 k^{2}}{27}$ and $|X \cap B|>\frac{2 k^{2}}{27}$. Then the number of $(X, \bar{X})$-type edges not in $A$ is at least $4 k^{4} / 729$.

Proof. Let $d$ denote $9|X \cap B| / k^{2}$. Then, $|E(X \cap B, \bar{X} \cap B)| \geq \frac{d(1-d) k^{4}}{81}$. Also, $\mid E(X \cap B, \bar{X} \cap$ $A) \mid \geq \frac{d k^{2}}{9} \cdot\left(\frac{k^{2}}{9}-|\bar{X} \cap A|\right) \geq \frac{d k^{2}}{9} \cdot \frac{k^{2}}{27}=\frac{d k^{4}}{243}$. The sum of these items is $k^{4} \frac{d+3 d(1-d)}{243} \geq \frac{4 k^{4}}{729}$.

Theorem 8. Let $k, n$ be such that 3 divides $k$ and $k$ divides $3 n$. The following is an isoperimetric function for $H(n, k)$ :

$$
f(x)= \begin{cases}\frac{k^{2} x}{27} & \text { if } 0 \leq x \leq \frac{k^{2}}{144} \\ \frac{k^{3} \sqrt{x}}{324} & \text { if } \frac{k^{2}}{144} \leq x \leq n^{2} / 2 .\end{cases}
$$

Proof. We make a $(3 n / k) \times(3 n / k)$ grid $G^{\prime}$ by cutting the original grid $G$ into $(k / 3) \times(k / 3)$ squares. Let us be given a set $X$ of vertices of $H(n, k),|X|=x\left(0 \leq x \leq n^{2} / 2\right)$. We have to find $f(x)$ edges in $H(n, k)$ leaving the set $X$. Every $v \in X$ falls into some $(3 n / k) \times(3 n / k)$ square in $G^{\prime}$, we call this square $S_{v}$. Set the density of the square $S_{v}$ as $d\left(S_{v}\right)=\left|X \cap S_{v}\right| /\left|S_{v}\right|=9\left|X \cap S_{v}\right| / k^{2}$. We distinguish cases:

(i) $v$ is small vertex, $\left(S_{v}\right.$ is a small square) if $d\left(S_{v}\right)<2 / 3$

(ii) $v$ is large vertex, $\left(S_{v}\right.$ is a large square) if $2 / 3 \leq d\left(S_{v}\right)$.

Let $x_{S}$ and $x_{L}$ denote the number of small (large) vertices of $X$. We have $x=x_{S}+x_{L}$. It follows from Lemma 3 that the number of $(X, \bar{X})$ type edges inside the small squares is at least $\frac{k^{2}\left(x-x_{L}\right)}{27}$.

Let us estimate the number of large squares of $G^{\prime}$. Clearly their number $n^{\prime}$ falls between $x_{L} /(k / 3)^{2}$ and $3 x_{L} /\left[2(k / 3)^{2}\right]$. According to the isoperimetric inequality for grids (26), the number of (small square, large square) pairs that share a side is at least $\sqrt{2 \min \left\{n^{\prime},(3 n / k)^{2}-n^{\prime}\right\}} \geq(3 / k) \sqrt{x_{L}}$. As before, we get

$$
|E(X, \bar{X})| \geq \frac{k^{2}\left(x-x_{L}\right)}{27}+\frac{k^{3} \sqrt{x_{L}}}{243} \geq \min \left(\frac{k^{2} x}{27}, \frac{k^{3} \sqrt{x}}{324}\right),
$$

and so we arrived at (35).

We remark here about the tightness of the new isoperimetric inequalities studied in this Section. Theorem 6 is tight within a multiplicative factor of 3 . To see this, consider a cut with a vertical line through $k$ horizontal edges of the rhombus of hexagons, as the rhombus of hexagons is drawn as in Fig. 1. This cut separates $k^{2}-1$ vertices from the rest. For Theorems 7 and 8 , an $\sqrt{x} \times \sqrt{x}$ subgrid shows the tightness of the isoperimetric functions within a constant multiplicative factor.

\section{Specific Results}

\subsection{Warm-Up}

The outerplanar crossing number of $K_{n}$ is exactly $\left(\begin{array}{l}n \\ 4\end{array}\right)$. This is a nice exercise that can be found in many texts in a somewhat different formulation. Let us see what kind of lower 
bound our method can show. By (24), $f(x)=x(n-x), f^{\prime \prime}(x)=-2, s=\lfloor n / 2\rfloor-3$, and $\kappa\left(f, s_{0}, s\right)=\frac{4}{3}+o(1)$ by $s_{0}=n^{1 / 3}$. Theorem 4 yields $\nu_{1}\left(K_{n}\right) \geq-\frac{n}{8}\left(\frac{4}{3}+o(1)\right) \int_{1}^{\lfloor n / 2\rfloor-4} x(n-$ $x)(-2) d x=\frac{n^{4}}{36}(1+o(1)$ ), which is $2 / 3$ of the truth. (On the other hand, Theorem 2 would yield only $\nu_{1}\left(K_{n}\right) \geq \frac{n^{4}}{48}$.)

\subsection{Estimating with Edge-Forwarding Index}

Theorem 9. For any connected graph $G$, we have

$$
\nu_{1}(G) \geq \frac{n^{4}(1-o(1))}{9 \pi^{2}(G)}-\frac{1}{2} \sum_{v} d_{v}^{2} .
$$

Note that the isoperimetric function (31) is only by a multiplicative factor of $\frac{2}{\pi(G)}$ off from the isoperimetric function (24) used in subsection 6.1. The multiplicative factor squares in that calculation, since we multiply with the second derivative. Therefore, we have for free from the calculations in subsection 6.1 that $\nu_{1}(G) \geq \frac{n^{4}}{36}(1+o(1))\left(\frac{2}{\pi(G)}\right)^{2}$, and Theorem 9 follows.

Note that exact values or good upper bounds for edge forwarding indices are known for many graph families, e.g. for the de Bruijn, Kautz, Butterfly and Cube-Connected-Cycles graphs $[8,16,17]$, also for the star and complete transposition graphs [7] and, in general, for edge symmetric Cayley graphs $[16,17]$.

For comparison, let us see what kind of lower bound can be provided for $\operatorname{cr}(G)$ in terms of $\pi(G)$. Theorem 3.11 in [13] easily adapts to embedding of multigraphs. Embed $2 K_{n}$ into $G$, where $2 K_{n}$ denotes the complete graph on $n$ vertices, where every edge comes with multiplicity two. Theorem 3.11 provides

$$
\operatorname{cr}(G) \geq \frac{\operatorname{cr}\left(2 K_{n}\right)}{\pi^{2}(G)}-\text { error term }
$$

However, it is well known that making $k$ copies of all edges of a graph $H$ to make multigraph $k H$, one has $\operatorname{cr}(k H)=k^{2} \operatorname{cr}(H)$, see [19]. Using this, (38) yields

$$
\operatorname{cr}(G) \geq \frac{4 \operatorname{cr}\left(K_{n}\right)}{\pi^{2}(G)}-\text { error term. }
$$

It is known that $\operatorname{cr}\left(K_{n}\right) \leq(1+o(1)) \frac{n^{4}}{64}$ and equality is conjectured here [20]. Therefore, the lower bound for $\nu_{1}$ in terms of edge forwarding index (37) is always better than the the lower bound for cr in terms of edge forwarding index (39).

\subsection{Hypercubes and Powers of Complete Graphs}

Theorem 10. For the outerplanar crossing number of the $n$-dimensional hypercube $\nu_{1}\left(Q_{n}\right)$, we have $(.1914+o(1)) \cdot 4^{n} \leq \nu_{1}\left(Q_{n}\right) \leq .5 \cdot 4^{n}$. 
Proof. Recall from [14] the drawing providing the upper bound. The drawing was constructed in the following way: put the vertices of $Q_{n}$ on the spine of a one-page book according to the Gray-code order. Draw the edges in one page in the usual recursive way. The number of crossings in the drawing is bounded from above by $4^{n} / 2$. By $(25), f(x)=x\left(n-\log _{2} x\right), f^{\prime}(x)=n-\log _{2} x-\frac{1}{\ln 2}, f^{\prime \prime}(x)=-\frac{1}{x \ln 2}$. Note that $f^{\prime}(x)>0$ only till $\frac{2^{n}}{e}$, so $f$ is not increasing on the entire interval $\left[0, \frac{|V(G)|}{2}-1\right]$; in other words Theorem 2 does not apply. However, Theorem 3 still applies. We choose $s=2^{n} / e$. One obtains $\kappa\left(f, s_{0}, s\right) \geq 1-o(1)$ by $s_{0}=n^{1 / 3}$. Theorem 4 yields $\nu_{1}\left(Q_{n}\right) \geq$ $(1-o(1)) \frac{2^{n}}{8} \int_{1}^{2^{n} / e-2} \frac{1}{(x+3) \ln 2} x\left(n-\log _{2} x\right) d x=4^{n} \frac{1}{4 e(\ln 2)^{2}}(1+o(1))$, which is more than $(.1914+o(1)) \cdot 4^{n}$.

The best lower bound for the crossing number of the hypercube is $\operatorname{cr}\left(Q_{n}\right) \geq \frac{1}{20}(1-$ $o(1)) 4^{n}[18]$. This lower bound applies to $\nu_{1}\left(Q_{n}\right)$, but we are not aware of any improvement for $\nu_{1}$. Analogues of Theorem 10 can be proved for $K_{p}^{n}\left(\right.$ as $\left.Q_{n}=K_{2}^{n}\right)$, using isoperimetric function (28) instead of (25)

\subsection{Powers of Petersen Graphs}

The isoperimetric function $f_{1}(x)$ in (29) is only a multiplicative factor $\frac{2}{5^{n}}$ off from the isoperimetric function of the complete graph $K_{5^{n}}$. Therefore, the calculations in subsection 6.1 apply and we obtain $\nu_{1}\left(P^{n}\right) \geq \frac{5^{4 n}}{36} \cdot\left(\frac{2}{5^{n}}\right)^{2}(1-o(1))=\left(\frac{1}{9}-o(1)\right) 5^{2 n} \geq .1111 \cdot 5^{2 n}$. The isoperimetric function $f_{2}(x)$ in $(30)$ is rather similar to the isoperimetric function (25). $f_{2}(x)$ increases till $5^{n} / e$, and like in the proof of Theorem 10, we can take $s_{0}=n^{1 / 3}$, $s=5^{n} / e$, and obtain $\kappa=1$. A calculation similar to the proof of Theorem 10 shows that

$\nu_{1}\left(P^{n}\right) \geq \frac{5^{2 n}}{e(\ln 5)^{2}}(1-o(1)) \geq .1420 \cdot 5^{2 n}$. The conclusion is, that the use of $f_{2}(x)$, which is not increasing till $5^{n} / 2$, yields better results. The hypercube is not the only example where we have to deal with isoperimetric functions taking maximum before the middle, since $K_{p}^{n}$ and $f_{2}(x)$ for $P^{n}$ behaves similarly. However, the exact isoperimetric function of $P^{n}$ is not known. The "natural" idea for improvement taking $f_{3}(x)=\max \left(f_{1}(x), f_{2}(x)\right)$ fails, since this function is not concave down (see Fig. 5 in [21]). We have no idea how to find an $f_{4}(x) \leq f_{3}(x)$ that satisfies the conditions of Theorem 4 and maximizes the lower bound (14). Such an $f_{4}(x)$ might provide a better lower bound for $\nu_{1}$ than any of $f_{1}(x)$, $f_{2}(x)$.

\subsection{Theorem 3 is stronger than Theorem 2}

As we have seen in the case of the hypercube, unlike Theorem 2, Theorem 3 remains applicable when the isoperimetric function is not increasing till $\left\lfloor\frac{\mid V(G)\rfloor}{2}\right\rfloor-1$. When both theorems are applicable, one cannot expect more than a multiplicative constant gain by using Theorem 3, though, as we have seen above, we can obtain such a gain. In the two applications above, the optimal choice of $s$ was always the maximum possible. A natural problem is the following: are there examples in which the optimal choice of $s$ is not the maximum possible allowed by the conditions? 
Consider the rectangular grid graph $P_{n} \times P_{k}$ with $k=\lfloor n \sqrt{\ln n}\rfloor$. Simple calculation based on (27) shows, that for any sufficiently small $\epsilon>0$ the choice $s=\left\lfloor(1-\epsilon) n^{2} / 2\right\rfloor$ beats $s=\lfloor n k / 2\rfloor-1$ in Theorem 3. Indeed, whatever is our choice for $s, \Delta^{2} f(j)=0$ for any $j>n^{2} / 2$. Approximating the summation with integral in (4), we have

$$
\nu_{1}\left(P_{n} \times P_{k}\right) \geq-\kappa\left(f, s_{0}, s\right)(1-o(1)) \cdot \frac{n k}{8} \int_{1}^{\min \left(s, n^{2} / 2\right)} f(x) f^{\prime \prime}(x) d x-\frac{1}{2} \sum_{v \in V} d_{v}^{2} .
$$

If $s=\lfloor n k / 2\rfloor-1$, we have to take $\kappa=1$, since some $m_{f, s}(l)$ values in $(2)$ are equal to 1 (say $\left.l=2 n^{2}\right)$, but $\kappa \geq 1$. However, for $s=\left\lfloor(1-\epsilon) n^{2} / 2\right\rfloor$ and any $s_{0} \rightarrow \infty$, we can take $\kappa=\frac{1}{\sqrt{1-2 \epsilon}}$, which is realized by $f(l) / f(s)=n / \sqrt{2 s}$ for any $l>2 s$, according to (27). Our claim boils down to the comparison of the integrals

$$
\frac{1}{\sqrt{1-2 \epsilon}} \int_{1}^{(1-\epsilon) n^{2} / 2} \frac{d x}{\sqrt{2} x} \text { and } \int_{1}^{n^{2} / 2} \frac{d x}{\sqrt{2} x} .
$$

The first integral is asymptotically bigger as $n \rightarrow \infty$, and this confirms our claim. We still have to show that we can select an $s_{0} \rightarrow \infty$ for $s=\left\lfloor(1-\epsilon) n^{2} / 2\right\rfloor$ so that we satisfy (3). We claim that any $s_{0}=\sqrt{\ln n}$ suffices. We have

$$
f\left(s_{0}\right)|E(G)|<\sqrt{2 s_{0}} \cdot 2 n k=2 s_{0} n^{2} \ln n=2 n^{2}(\ln n)^{3 / 4} .
$$

On the other hand, $P_{n} \times P_{k}$ contains a $P_{n} \times P_{n}$ subgraph. Observe that for $x \leq n^{2} / 2$ the same $f(x)=\sqrt{2 x}$ is isoperimetric function for both $P_{n} \times P_{k}$ and $P_{n} \times P_{n}$. We already know [15] for $P_{n} \times P_{n}$ that

$$
\sum_{e \in E\left(P_{n} \times P_{n}\right)} f(l(e))=\Omega\left(n^{2} \ln n\right) .
$$

Formula (42) must be valid if the summation is extended for $e \in E\left(P_{n} \times P_{k}\right)$. Furthermore, (42) must still be valid, if restricted for edges of length at least $s_{0}$ :

$$
\sum_{e \in E\left(P_{n} \times P_{k}\right): l(e) \geq s_{0}} f(l(e))=\Omega\left(n^{2} \ln n\right) .
$$

The reason is that the last two summations differ by at most $\left|E\left(P_{n} \times P_{k}\right)\right| \leq 2 n k=$ $2 n^{2} \sqrt{\ln n}$ terms, and each term is at most $f\left(s_{0}\right)=\sqrt{2}(\ln n)^{1 / 4}$, and hence the two summations differ by $O\left(n^{2}(\ln n)^{3 / 4}\right)=o\left(n^{2} \ln n\right)$. Finally, observe that (41) and (43) together prove the second case of (3) for the graph $P_{n} \times P_{k}$.

\subsection{Rhombus of Hexagons}

In [15] we did not establish an isoperimetric function for the rhombus of hexagons, instead, we used an ad hoc technique (a variant of embedding) to show that the outerplanar crossing number of the rhombus of hexagons is $\Omega\left(n^{2} \log n\right)$. Based on the isoperimetric function in (32), Theorem 2 or 3 yields this result immediately. 


\subsection{Some Dense Geometric Graphs}

Consider $G(n, k)$ the graph defined in Section 5. We claim that $\operatorname{cr}(G(n, k))=\Theta\left(k^{4} n^{2}\right)$ for $1 \leq k \leq n$. The upper bound follows from a natural grid-like drawing, and the lower bound follows from a bisection width argument (see [11]), since the $(1 / 3-2 / 3)$ bisection width of $G(n, k)$ is at least $\Omega\left(k^{2} n\right)$, which immediately follows from Theorem 7 , for any $k \leq n$. We show here that $\nu_{1}(G(n, k))$ is bigger by a $\log n$ factor than $\operatorname{cr}(G(n, k))$.

Theorem 11. For every $\epsilon>0$ and $k \leq n^{1-\epsilon}$, as $n \rightarrow \infty$, we have

$$
\nu_{1}(G(n, k))=\Theta\left(k^{4} n^{2} \log n\right) .
$$

Proof. The upper bound follows from Theorem 1. The lower bound follows from Theorem 2 and the isoperimetric inequality in Theorem 7, (33).

Consider $H(n, k)$ the graph defined in section 5. It is known that $\operatorname{cr}(H(n, k))=$ $\Theta\left(n^{2} k^{6}\right)$. The upper bound follows from the natural straight line drawing of the graph, with infinitesimally small modification, to make sure that edges do not pass through vertices. The lower bound follows from a bisection width argument (see [11]), since the $(1 / 3-2 / 3)$ bisection width of $H(n, k)$ is at least $\Omega\left(k^{3} n\right)$, which immediately follows from Theorem 8 , for any $k \leq n$. We show here that $\nu_{1}(H(n, k))$ is bigger than $\operatorname{cr}(H(n, k))$ by a $\log n$ factor.

Theorem 12. For every $\epsilon>0$ and $k \leq n^{1-\epsilon}$, as $n \rightarrow \infty$, we have

$$
\nu_{1}(H(n, k))=\Theta\left(k^{6} n^{2} \log n\right) .
$$

Proof. The upper bound follows from Theorem 1. The lower bound follows from Theorem 2 and the isoperimetric inequality in Theorem 8, (35).

Remark 1. Note that Theorems 11, 12 fail for $k=n$, and therefore a condition like $k \leq n^{1-\epsilon}$ is necessary.

\subsection{Linear Arrangement in Norms less than 1}

Observe that $F(x)=x^{\alpha}$ with $0<\alpha<1$ satisfies all the requirements of Theorem 5. For simplicity, we abbreviate $L_{F}^{o}(G)$ by $L_{\alpha}^{o}(G)\left(L_{F}(G)\right.$ by $\left.L_{\alpha}(G)\right)$ for $F(x)=x^{\alpha}$. We show as an application

Theorem 13. For the grid $G=P_{n} \times P_{n}$, we have

$$
L_{\alpha}(G)= \begin{cases}\Theta\left(n^{2}\right) & \text { if } 0<\alpha<1 / 2 \\ \Theta\left(n^{2} \log n\right) & \text { if } \alpha=1 / 2 \\ \Theta\left(n^{2 \alpha+1}\right) & \text { if } 1 / 2<\alpha<1 .\end{cases}
$$


Proof. Apply the isoperimetric inequality (26) and formula (22) from Theorem 5 to obtain the lower bound for $L_{\alpha}^{o}(G)$, and hence for $L_{\alpha}(G)$. The upper bound is given by the following recursive layout of the grid. Divide the grid $P_{n} \times P_{n}$ into four subgrids $P_{\frac{n}{2}} \times P_{\frac{n}{2}}$ by removing $2 n$ edges, lay down the the subgrids recursively and add the $2 n$ edges back. We get the recurrence relation: $L_{\alpha}\left(P_{n} \times P_{n}\right) \leq 4 L_{\alpha}\left(P_{\frac{n}{2}} \times P_{\frac{n}{2}}\right)+2 n^{2 \alpha+1}$. The solution of the recurrence relation is

$$
L_{\alpha}\left(P_{n} \times P_{n}\right)= \begin{cases}O\left(n^{2}\right) & \text { if } 0<\alpha<1 / 2 \\ O\left(n^{2} \log n\right) & \text { if } \alpha=1 / 2 \\ O\left(n^{2 \alpha+1}\right) & \text { if } 1 / 2<\alpha<1\end{cases}
$$

Acknowledgement. We thank Farhad Shahrokhi for discussions about the outerplanar crossing numbers, and Hua Wang for his careful reading of the manuscript.

\section{References}

[1] Bezrukov, S., Schroeder, U.-P., The cyclic wirelength of trees, Discrete Appl. Math. 87 (1998), 275-277.

[2] Bollobás, B., Leader, I., Edge-isoperimetric inequalities in the grid, Combinatorica, 11 (1991), 299-314.

[3] Ching-Jung Guu, The circular wirelength problem for the hypercube, Ph.D. Thesis, University of California, Riverside, 1997.

[4] Chung, F.R.K., Füredi, Z., Graham, R.L., Seymour, P.D.: On induced subgraphs of the cube, J. Comb. Theory A-49 (1988), 180-187.

[5] Crimmins, R., T., Horwitz, H.M., Palermo, C. J., Minimization of mean-square error for data transmitted via group codes, IEEE Trans. Information Theory 15 (1969) $72-78$.

[6] Di Battista, J., Eades, P., Tamassia, R., Tollis, I. G., Graph Drawing. Algorithms for the Visualization of Graphs, Prentice Hall Inc., Upper Saddle River, NJ, 1999, $432 \mathrm{pp}$.

[7] Gauyacq, G., Forwarding index of cayley graphs and star graphs, Discrete Applied Mathematics 80 (1997), 149-160.

[8] Heydemann, M.C., Meyer, J.C., Sotteau, D., On forwarding indices of networks, Discrete Applied Mathematics 23 (1989), 103-123.

[9] Juvan, M., Mohar, B., Optimal linear labelings and eigenvalues of graphs, Discrete Appl. Math. 36 (1992) 153-168. 
[10] Kainen, P.C., The book thickness of a graph II, Congressus Numerantium, 71 (1990), $121-132$.

[11] Pach, J., Shahrokhi F., and Szegedy, M., Applications of crossing numbers, in: Proc. 10-th Annual ACM Symposium on Computational Geometry (ACM, New York, 1994); and Algorithmica 16 (1996) 111-117.

[12] Purchase, H., Which aesthetic has the greatest effect on human understanding?, in: Proc. Symposium on Graph Drawing, GD'97, Lecture Notes in Computer Science 1353 Springer, 1997, 248-261.

[13] Shahrokhi, F., Sýkora, O., Székely, L. A., and Vrťo, I., Crossing numbers: bounds and applications, in: Intuitive Geometry, eds. I. Bárány and K. Böröczky, Bolyai Society Mathematical Studies 6, János Bolyai Mathematical Society, Budapest, 1997, 179206.

[14] Shahrokhi, F., Sýkora, O., Székely, L.A., Vrto, I., Book embeddings and crossing numbers, in: Proc. 20th Intl. Workshop on Graph-Theoretic Concepts in Computer Science, WG'94, Lecture Notes in Computer Science 903, Springer, 1995, 256-268.

[15] Shahrokhi, F., Sýkora, O., Székely, L.A., Vrto, I., Towards the theory of convex crossing numbers, In: Towards a Theory of Geometric Graphs, ed. J. Pach, Contemporary Mathematics, 342 American Mathematical Society, Providence 2004, 249-258.

[16] Shahrokhi, F., Székely, L.A., An algebraic approach to the uniform concurrent multicommodity flow problem: Theory and applications, Technical Report CRPDC-91-4, DCS, Uni. North Texas, Denton, 1991.

[17] Shahrokhi., F., Székely, L. A., Constructing integral uniform flows in symmetric networks and application to the forwarding index problem, Discrete Applied Math. 108 (2001), 175-191.

[18] Sýkora, O., Vrto, I., On the crossing number of hypercubes and cube conected cycles, BIT 33 (1993), 232-237.

[19] Székely, L. A., Crossing numbers and hard Erdős problems in discrete geometry, Combinatorics, Probability, and Computing 6(3)(1997), 353-358.

[20] Székely, L.A., A successful concept for measuring non-planarity of graphs: the crossing number, Discrete Math. 276 (2003), 1-3, 331-352.

[21] Tillich, J.-P., Edge isoperimetric inequalities for product graphs, Discrete Mathematics 213 (2000), 291-320. 